\title{
Electron-plasmon model in the electron liquid theory
}

\author{
M.V.Vavrukh, S.B.Slobodyan \\ Ivan Franko National University of Lviv, \\ Chair for Astrophysics, \\ 8 Kyrylo and Methodiy Str., \\ 79005 Lviv, Ukraine
}

Received June 17, 2004, in final form February 16, 2005

\begin{abstract}
Here we propose an accurate approach to the description of the electron liquid model in the electron and plasmon terms. Our ideas in the present paper are close to the conception of the collective variables which was developed in the papers of Bohm and Pines. However we use another body of mathematics in the transition to the expanded space of variable particles and plasmons realized by the transition operator. It is evident that in the Random Phase Approximation (RPA), the model which consists of two interactive subsystems of electrons and plasmons is equivalent to the electron liquid model with Coulomb interaction.
\end{abstract}

Key words: electron liquid, plasmon oscillation, correlation energy, transition operator, structural factor

PACS: 05.30.Fk

\section{Introduction}

Co-existence of the collective and individual motions is the characteristic feature of the systems with the collective electrons (the metals, the superconductors, degenerate semiconductors) which conditioned the long-range character of the Coulomb potential. This circumstance is the main basis for the formulation of a simple approach in the metal theory. This approach is based on the conception of collective description of interelectron interactions. One of the variants of this approach was developed in the papers [1-6] (see also [7]; almost all the papers in this field are indicated). Since the plasmons are the well-determined excitations only at small values of the wave vector $\mathbf{q}$, in these papers there were introduced the collective variables for the region $0 \leqslant|\mathbf{q}| \leqslant k_{c}$ where $k_{c}$ has the order of magnitude $k_{\mathrm{F}}=\left(3 \pi^{2} N\right)^{\frac{1}{3}} V^{-\frac{1}{3}}$. In order to conserve the number of degrees of freedom, subsidiary conditions are put on the wave function of the system [5]. As a rule in the cited papers the ground state system was examined. One of the main tasks was to investigate the spectrum of the 
plasmon oscillations. To this end, a series of canonical transformations was used. As a result, the developed formalism has assumed an approximate character. In our opinion, the approach of Bohm and Pines was displaced from the metal theory by a more formal approach of the summation diagram series of the ordinary perturbation theory which was started by the paper of Gell-Mann and Brueckner [8]. This approach complemented by the local-field conception is considered to be general in the modern metal theory. The results obtained by this approach are far better than the results obtained within the framework of the collective description. For example, let us examine the correlation energy being the characteristic of the electron liquid model weakly sensitive to the standard approximations. The correlation energy that was calculated in paper [6] differs from the results of Monte-Carlo method [9] (which are considered to be standard) as well as from many analitical methods (see for example [10-14]) developed within the framework of the ordinary perturbation theory. Table 1 shows the dependence of correlation energy of the electron liquid model (in the rydberg per electron) on the non-ideality parameter $r_{s}=a_{0}^{-1}(3 V)^{\frac{1}{3}}(4 \pi N)^{-\frac{1}{3}}$ where $a_{0}$ is the Bohr radius.

Table 1. Correlation energy of the electron liquid model $\left(-10^{3} \varepsilon\left(r_{s}\right)\right)$.

\begin{tabular}{|c|c|c|c|c|c|c|c|}
\hline$r_{s}$ & 1 & 2 & 3 & 4 & 5 & 6 & 10 \\
\hline RPA & 157.6 & 123.6 & 105.5 & 93.6 & 84.95 & 78.2 & 61.3 \\
\hline NP & 115 & 94 & 81 & 72 & 65 & 60 & \\
\hline TW & 134 & 95 & 79 & 68 & 61 & & \\
\hline VS & 112 & 89 & 75 & 65 & 58 & 52 & \\
\hline EZ & 122.0 & 90.4 & 73.8 & 63.4 & 56.0 & 50.5 & \\
\hline STLS & 124 & 92 & 75 & 64 & 56 & 50 & 36 \\
\hline IU & 117.4 & 86.9 & 71.1 & 61.0 & 53.8 & 48.3 & 35.0 \\
\hline CA & 121.1 & 90.8 & 74.96 & 64.7 & 57.4 & 51.8 & 38 \\
\hline
\end{tabular}

$[\mathrm{NP}]-[6] ;[\mathrm{TW}]-[11] ;[\mathrm{VS}]-[12] ;[\mathrm{EZ}]-[13] ;$

$[\mathrm{STLS}]-[10] ;[\mathrm{IU}]-[14] ;[\mathrm{CA}]-[9]$.

Evidently, the collective description of interelectron interactions has preference to the methods of the ordinary perturbation theory, especially in the strong nonideality region. It is caused by the fact that in the collective description, the system of free electrons and non-interaction plasmons is used in the role of zero approximation rather than the ideal electron system. The conception of collective description has a further prospective development. The variant which was developed in papers $[1-6]$ is one of the possible variants. There exists another variant of collective description which was developed in the papers [15-18]. In this variant, together with introducing the expanded space of individual and collective coordinates, transformation of displacements in the statistical operator is used. Thus we take the infinite system of non-linear integro-differential equations. This makes this method an approximate one. In the method of displacements and collective variables, the transition to ex- 
panded space is realized strictly by means of the transition function as opposed to Bohm-Pines approach. Both approaches have much in common. The characteristic feature of both approaches is the absence of divergent diagrams as opposed to the standard methods of the perturbation theory.

In the present paper we propose the variant of collective description of interactions in the electron liquid model which differs from Bohm-Pines variant as well as from the variant of displacements and collective variables. We start with the secondary quantization representation. Then we transit to expanded space by means of the transition operator which was first introduced in the paper [19]. The collective variables are an intermediate element. They are used for introduction of operators of the creation and destruction of plasmons. Partition function of the model in the electron and plasmon terms does not have any approximations. The perturbation theory relatively to the electron-plasmon interaction is built in terms of the $n$-particles dynamic correlation function. The short-range interelectron interactions are taken into account in the local-field approximation.

\section{General relations}

Let us consider Hamiltonian of the electron liquid model in the secondary quantization representation on the plane wave base

$$
\begin{aligned}
& \widehat{H}=\widehat{H}_{0}+\widehat{V}, \quad \widehat{H}_{0}=\sum_{\mathbf{k}, s} \varepsilon_{\mathbf{k}} a_{\mathbf{k}, s}^{+} a_{\mathbf{k}, s}, \\
& \widehat{V}=(2 V)^{-1} \sum_{\mathbf{q} \neq 0} V_{\mathbf{q}} \sum_{\mathbf{k}_{1}, \mathbf{k}_{2}} \sum_{s_{1}, s_{2}} a_{\mathbf{k}_{1}+\mathbf{q}, s_{1}}^{+} a_{\mathbf{k}_{2}-\mathbf{q}, s_{2}}^{+} a_{\mathbf{k}_{2}, s_{2}} a_{\mathbf{k}_{1}, s_{1}},
\end{aligned}
$$

where $\varepsilon_{\mathbf{k}}=\hbar^{2} \mathbf{k}^{2} / 2 m, V_{\mathbf{q}}=4 \pi e^{2} \mathbf{q}^{-2}, V$ - volume system. The antisymmetric wave function of the system $\Psi$ depends not only on individual but also on collective variables due to interaction between electrons. The wave functions of this type were used in a number of papers (see [20-22]). In the secondary quantization the wave function can be written as follows: $\Psi \equiv \Psi\left(a_{\mathbf{k}, s} \mid \widehat{\rho}_{\mathbf{q}}\right)$, where $\widehat{\rho}_{\mathbf{q}}=\sum_{\mathbf{k}, s} a_{\mathbf{k}+\mathbf{q}, s}^{+} a_{\mathbf{k}, s}$ is Fourier representation of the operator of the electron density. Here $a_{\mathbf{k}, s}$ and $\hat{\rho}_{\mathbf{q}}$ are not independent.

Let us transit individual and collective variables to the expanded space by means of transition operator [19]

$$
\widehat{J}(\rho, \widehat{\rho})=\prod_{C_{\mathbf{q}}} \delta\left(\rho_{\mathbf{q}}-\widehat{\rho}_{\mathbf{q}}\right)=\int(\mathrm{d} \omega)\left\{2 \pi \mathrm{i} \sum_{\mathbf{q} \in C_{\mathbf{q}}} \omega_{\mathbf{q}}\left(\rho_{\mathbf{q}}-\widehat{\rho}_{\mathbf{q}}\right)\right\},
$$

where $\omega_{\mathbf{q}}$ is a variable, which is conjugated to $\rho_{\mathbf{q}},(\mathrm{d} \omega)=\prod_{C_{\mathbf{q}}}\left(\mathrm{d} \omega_{\mathbf{q}}\right)$. The range of wave vectors $C_{\mathbf{q}}$ includes one-half of all the vectors from the sphere at radius $q_{0}$, for example

$$
0<|\mathbf{q}| \leqslant q_{0}, \quad q_{z}>0
$$


The transition operator is a quantum analogue of the transition function of the classical statistics $[23,24]$. Any operator $\widehat{f}(\widehat{\rho})$ represented in $\widehat{\rho}_{\mathbf{q}}$ term has the following representation in the collective variables:

$$
\widehat{f}(\widehat{\rho})=\int(\mathrm{d} \rho) \widehat{J}(\rho, \widehat{\rho}) \widehat{F}(\rho), \quad(\mathrm{d} \rho) \equiv \prod_{C_{\mathbf{q}}} \mathrm{d} \rho_{\mathbf{q}}
$$

Relationships

$$
\begin{aligned}
\widehat{J}\left(\rho, \rho^{\prime}\right) \widehat{J}\left(\rho^{\prime}, \widehat{\rho}\right) & =\widehat{J}(\rho, \widehat{\rho}) \prod_{C_{\mathbf{q}}} \delta\left(\rho_{\mathbf{q}}^{\prime}-\rho_{\mathbf{q}}\right) \\
\operatorname{Sp}\left\{\widehat{J}(\rho, \widehat{\rho}) \widehat{J}\left(\rho^{\prime}, \widehat{\rho}\right)\right\} & =\prod_{C_{\mathbf{q}}} \delta\left(\rho_{\mathbf{q}}{ }^{\prime}-\rho_{\mathbf{q}}\right) \operatorname{Sp} \widehat{J}(\rho, \widehat{\rho})
\end{aligned}
$$

allow to find the representation of $\widehat{f}(\widehat{\rho})$ in the collective variables, namely

$$
\widehat{F}(\rho)=\{\operatorname{Sp} \widehat{J}(\rho, \widehat{\rho})\}^{-1} \operatorname{Sp}\{\widehat{f}(\widehat{\rho}) \widehat{J}(\rho, \widehat{\rho})\} .
$$

Similarly to the papers [1-6] the energy operator of the interelectron interections is divided into long-range and short-range terms and is written in the following form

$$
\frac{1}{2 V} \sum_{\mathbf{q} \in \mathbf{C}_{\mathbf{q}}} V_{\mathbf{q}}\left(\widehat{\rho}_{\mathbf{q}} \widehat{\rho}_{-\mathbf{q}}-\widehat{N}\right)+\frac{1}{2 V} \sum_{\mathbf{q} \notin C_{\mathbf{q}}} V_{\mathbf{q}} \sum_{\mathbf{k}_{1}, \mathbf{k}_{2}} \sum_{s_{1}, s_{2}} a_{\mathbf{k}_{1}+\mathbf{q}, s_{1}}^{+} a_{\mathbf{k}_{2}-\mathbf{q}, s_{2}}^{+} a_{\mathbf{k}_{2}, s_{2}} a_{\mathbf{k}_{1}, s_{1}},
$$

where $\widehat{N} \equiv \sum_{\mathbf{k}, s} a_{\mathbf{k}, s}^{+} a_{\mathbf{k}, s}$ is the operator of the number particles. The second term in the (6) describes the effective short-range interaction electrons with the potential

$$
V_{s}(\mathbf{r})=\frac{1}{V} \sum_{\mathbf{q} \notin C_{\mathbf{q}}} V_{\mathbf{q}} \exp (\text { iqr })=\frac{e^{2}}{r}\left\{1-\frac{2}{\pi} \operatorname{Si}\left(q_{0} r\right)\right\}
$$

$(\operatorname{Si}(x)$ is a sine integral $[25])$.

Correspondingly to formula (3) any wave function $\Psi\left(a_{k} \mid \widehat{\rho}\right)$ the reprezentation in the expanded space $\Psi\left(a_{k} \mid \rho\right)$ can be compared,

$$
\Psi\left(a_{k} \mid \widehat{\rho}\right)=\int(\mathrm{d} \rho) \widehat{J}(\rho, \widehat{\rho}) \Psi\left(a_{k} \mid \rho\right)
$$

Let us calculate the operation $\exp (-\beta \widehat{H})$ to the wave function $\Psi\left(a_{k} \mid \widehat{\rho}\right)$ :

$$
\begin{aligned}
\exp (-\beta \widehat{H}) \Psi\left(a_{k} \mid \widehat{\rho}\right)= & \int(\mathrm{d} \rho)(\mathrm{d} \omega) \exp \left(-2 \pi \mathrm{i} \sum_{\mathbf{q} \in C_{\mathbf{q}}} \omega_{\mathbf{q}} \widehat{\rho}_{\mathbf{q}}\right) \\
& \times \exp \left(-\beta \widehat{H}_{\omega}\right) \exp \left(2 \pi \mathrm{i} \sum_{\mathbf{q} \in C_{\mathbf{q}}} \omega_{\mathbf{q}} \rho_{\mathbf{q}}\right) \Psi\left(a_{k} \mid \rho\right) .
\end{aligned}
$$


Here $\beta=\left(k_{\mathrm{B}} T\right)^{-1}$ is the inverse temperature,

$$
\begin{aligned}
\widehat{H}_{\omega} & =\exp \left(2 \pi \mathrm{i} \sum_{\mathbf{q} \in C_{\mathbf{q}}} \omega_{\mathbf{q}} \widehat{\rho}_{\mathbf{q}}\right) \widehat{H} \exp \left(-2 \pi \mathrm{i} \sum_{\mathbf{q} \in C_{\mathbf{q}}} \omega_{\mathbf{q}} \widehat{\rho}_{\mathbf{q}}\right) \\
& =\widehat{H}-2 \pi \mathrm{i} \sum_{\mathbf{q} \in C_{\mathbf{q}}} \omega_{\mathbf{q}}\left[\widehat{H}_{0}, \widehat{\rho}_{\mathbf{q}}\right]_{-}+\frac{(2 \pi \mathrm{i})^{2}}{2 !} \sum_{\mathbf{q}_{1}, \mathbf{q}_{2} \in C_{\mathbf{q}}} \omega_{\mathbf{q}_{1}} \omega_{\mathbf{q}_{2}}\left[\left[\widehat{H}_{0}, \widehat{\rho}_{\mathbf{q}_{1}}\right]_{-}, \widehat{\rho}_{\mathbf{q}_{2}}\right]_{-}
\end{aligned}
$$

Here is the explicit form of the commutators:

$$
\begin{aligned}
{\left[\widehat{H}_{0}, \widehat{\rho}_{\mathbf{q}}\right]_{-} } & =\varepsilon_{\mathbf{q}} \widehat{\rho}_{\mathbf{q}}+\frac{\hbar^{2}}{m} \widehat{f}_{\mathbf{q}} ; \quad \widehat{f}_{\mathbf{q}} \equiv \sum_{\mathbf{k}, s}(\mathbf{k}, \mathbf{q}) a_{\mathbf{k}+\mathbf{q}, s}^{+} a_{\mathbf{k}, s} ; \\
\frac{1}{2}\left[\left[\widehat{H}_{0}, \widehat{\rho}_{\mathbf{q}_{1}}\right]_{-}, \widehat{\rho}_{\mathbf{q}_{2}}\right]_{-} & =-\widehat{N}_{\mathbf{q}_{1}} \delta_{\mathbf{q}_{1}+\mathbf{q}_{2}, 0}+\frac{\hbar^{2}}{2 m}\left(\mathbf{q}_{1}, \mathbf{q}_{2}\right) \widehat{\rho}_{\mathbf{q}_{1}+\mathbf{q}_{2}} .
\end{aligned}
$$

Let us apply the identity

$$
\left\{2 \pi \mathrm{i} \omega_{\mathbf{q}}-\frac{\partial}{\partial \rho_{\mathbf{q}}}\right\} \exp \left(2 \pi \mathrm{i} \sum_{\mathbf{q} \in C_{\mathbf{q}}} \omega_{\mathbf{q}} \rho_{\mathbf{q}}\right)=0
$$

and integrating in parts over the variables $\rho_{\mathbf{q}}$ let us bring the equality (9) to the following form:

$$
\begin{aligned}
\exp (-\beta \widehat{H}) \Psi\left(a_{k} \mid \widehat{\rho}\right)= & \int(\mathrm{d} \rho) \widehat{J}(\rho, \widehat{\rho}) \exp \left(-\beta \widehat{H}_{1}\left(a_{k} \mid \rho\right)\right) \Psi\left(a_{k} \mid \rho\right) \\
\widehat{H}_{1}\left(a_{k} \mid \rho\right)= & \widehat{H}+\sum_{\mathbf{q} \in C_{\mathbf{q}}}\left(\varepsilon_{\mathbf{q}} \widehat{\rho}_{\mathbf{q}}+\frac{\hbar^{2}}{m} \widehat{f}_{\mathbf{q}}\right) \frac{\partial}{\partial \rho_{\mathbf{q}}}-\widehat{N} \sum_{\mathbf{q} \in C_{\mathbf{q}}} \varepsilon_{\mathbf{q}} \frac{\partial^{2}}{\partial \rho_{\mathbf{q}} \partial \rho_{-\mathbf{q}}} \\
& +\frac{\hbar^{2}}{2 m} \sum_{\mathbf{q}_{1}, \mathbf{q}_{2} \in C_{\mathbf{q}}}\left(\mathbf{q}_{1}, \mathbf{q}_{2}\right) \widehat{\rho}_{\mathbf{q}_{1}+\mathbf{q}_{2}} \frac{\partial^{2}}{\partial \rho_{\mathbf{q}_{1}} \partial \rho_{\mathbf{q}_{2}}}
\end{aligned}
$$

Here $\widehat{H}$ was defined by formula (1). According to (3), the substitution $\widehat{\rho}_{\mathbf{q}} \rightarrow \rho_{\mathbf{q}}$ in the operator $\widehat{H}_{1}\left(a_{k} \mid \rho\right)$ can be realized. Therefore the partition function of the model in the grand canonical ensemble has the following form

$$
Z=\mathrm{Sp} \exp \{-\beta(\widehat{H}-\mu \widehat{N})\}=\operatorname{Sp} \int(\mathrm{d} \rho) \widehat{J}(\rho, \widehat{\rho}) \exp \{-\beta(\widehat{\mathcal{H}}(a \mid \rho)-\mu \widehat{N})\}
$$

where the $\mu$ is the chemical potential variable, $\widehat{N}$ is the operator of the complete number of particles. The operator

$$
\begin{aligned}
\widehat{\mathcal{H}}(a \mid \rho)= & \widehat{H}_{s}+\sum_{\mathbf{q} \in C_{\mathbf{q}}}\left\{\left(\varepsilon_{\mathbf{q}} \rho_{\mathbf{q}}+\frac{\hbar^{2}}{m} \widehat{f}_{\mathbf{q}}\right) \frac{\partial}{\partial \rho_{\mathbf{q}}}-\widehat{N} \varepsilon_{\mathbf{q}} \frac{\partial^{2}}{\partial \rho_{\mathbf{q}} \partial \rho_{-\mathbf{q}}}+(2 V)^{-1} V_{\mathbf{q}}\left[\rho_{\mathbf{q}} \rho_{-\mathbf{q}}-\widehat{N}\right]\right\} \\
& +\frac{\hbar^{2}}{2 m} \sum_{\mathbf{q}_{1}, \mathbf{q}_{2} \in C_{\mathbf{q}}}\left(\mathbf{q}_{1}, \mathbf{q}_{2}\right) \rho_{\mathbf{q}_{1}+\mathbf{q}_{2}} \frac{\partial^{2}}{\partial \rho_{\mathbf{q}_{1}} \partial \rho_{\mathbf{q}_{2}}}
\end{aligned}
$$


is Hamiltonian of the system in the expanded space of individual and collective variables, $a_{k}$ and $\rho_{\mathbf{q}}$ are independent. The transition operator regulates the correlation between variables and provides the conservation of the number of the degree of freedom. The operator

$$
\widehat{H}_{s}=\widehat{H}_{0}+(2 V)^{-1} \sum_{\mathbf{q} \notin C_{\mathbf{q}}} V_{\mathbf{q}} \sum_{\mathbf{k}_{1}, \mathbf{k}_{2}} \sum_{s_{1}, s_{2}} a_{\mathbf{k}_{1}+\mathbf{q}, s_{1}}^{+} a_{\mathbf{k}_{2}-\mathbf{q}, s_{2}}^{+} a_{\mathbf{k}_{2}, s_{2}} a_{\mathbf{k}_{1}, s_{1}}
$$

is the Hamiltonian of the electron system with the short-range interaction.

Let us further consider the improved variant of calculation of the partition function using its cyclic transformation by virtue of the operator

$$
\widehat{W}(\widehat{\rho})=\frac{1}{2} \sum_{\mathbf{q} \in C_{\mathbf{q}}} w(\mathbf{q}) \widehat{\rho}_{\mathbf{q}} \widehat{\rho}_{-\mathbf{q}}
$$

where $w(\mathbf{q})$ is the unknown function which will be found later. Since $\widehat{W}(\widehat{\rho})$ commutates with the OPERATORS $\widehat{N}$ and $\widehat{V}$, then in the renormalized partition function

$$
Z=\operatorname{Sp}\left\{\mathrm{e}^{-\widehat{W}(\widehat{\rho})} \exp (-\beta[\widehat{H}-\mu \widehat{N}]) \mathrm{e}^{\widehat{W}(\widehat{\rho})}\right\}=\operatorname{Sp}\left\{\exp \left[-\beta\left(\widehat{H}_{W}-\mu \widehat{N}\right)\right]\right\}
$$

there appears renormalized Hamiltonian

$$
\begin{aligned}
\widehat{H}_{W}= & \widehat{H}_{0}+\widehat{V}+\widehat{K}+\widehat{L} ; \\
\widehat{K}= & {\left[\widehat{H}_{0}, \widehat{W}\right]_{-}=\sum_{\mathbf{q} \in C_{\mathbf{q}}} w(\mathbf{q})\left\{\varepsilon_{\mathbf{q}}\left[\widehat{\rho}_{\mathbf{q}} \widehat{\rho}_{-\mathbf{q}}-\widehat{N}\right]+\frac{\hbar^{2}}{m} \widehat{\rho}_{\mathbf{q}} \widehat{f}_{-\mathbf{q}}\right\} ; } \\
\widehat{L}= & \frac{1}{2}\left[\left[\widehat{H}_{0}, \widehat{W}\right]_{-}, \widehat{W}\right]_{-}=-\widehat{N} \sum_{\mathbf{q} \in C_{\mathbf{q}}} \varepsilon_{\mathbf{q}} w^{2}(\mathbf{q}) \widehat{\rho}_{\mathbf{q}} \widehat{\rho}_{-\mathbf{q}} \\
& +\frac{\hbar^{2}}{2 m} \sum_{\mathbf{q} 1, \mathbf{q}_{2} \in C_{\mathbf{q}}}\left(\mathbf{q}_{1}, \mathbf{q}_{2}\right) w\left(\mathbf{q}_{1}\right) w\left(\mathbf{q}_{2}\right) \widehat{\rho}_{\mathbf{q}_{1}} \widehat{\rho}_{\mathbf{q}_{2}} \widehat{\rho}_{-\mathbf{q}_{1}-\mathbf{q}_{2}} .
\end{aligned}
$$

The operation of $\exp \left(-\beta \widehat{H}_{W}\right)$ on function $\Psi\left(a_{k} \mid \widehat{\rho}\right)$ is calculated similarly to the operation $\exp (-\beta \widehat{H})$ (see expressions $(9)-(14)$ ). The analogue of the formula (14) is the following expression:

$$
\begin{gathered}
Z=\operatorname{Sp} \int(\mathrm{d} \rho) \widehat{J}(\rho, \widehat{\rho}) \exp \left\{-\beta\left(\widehat{\mathcal{H}}_{W}(a \mid \rho)-\mu \widehat{N}\right)\right\} \\
\widehat{\mathcal{H}}_{W}(a \mid \rho)=\widehat{H}_{s}-\widehat{N} \sum_{\mathbf{q} \in C_{\mathbf{q}}}\left(\frac{V_{\mathbf{q}}}{2 V}+w(\mathbf{q}) \varepsilon_{\mathbf{q}}\right) \\
+\sum_{\mathbf{q} \in C_{\mathbf{q}}}\left\{\frac{1}{2} \rho_{\mathbf{q}} \rho_{-\mathbf{q}}\left[\frac{V_{\mathbf{q}}}{V}+2 \varepsilon_{\mathbf{q}} w(\mathbf{q})(1-\widehat{N} w(\mathbf{q}))\right]-\widehat{N} \varepsilon_{\mathbf{q}} \frac{\partial^{2}}{\partial \rho_{\mathbf{q}} \partial \rho_{-\mathbf{q}}}\right. \\
\left.+\frac{\hbar^{2}}{m}\left[w(\mathbf{q}) \rho_{-\mathbf{q}}+\frac{\partial}{\partial \rho_{\mathbf{q}}}\right] \widehat{f}_{\mathbf{q}}+[1-2 \widehat{N} w(\mathbf{q})] \varepsilon_{\mathbf{q}} \rho_{\mathbf{q}} \frac{\partial}{\partial \rho_{\mathbf{q}}}\right\} \\
+\frac{\hbar^{2}}{2 m} \sum_{\mathbf{q}_{1}, \mathbf{q}_{2} \in C_{\mathbf{q}}}\left(\mathbf{q}_{1}, \mathbf{q}_{2}\right) \rho_{\mathbf{q}_{1}+\mathbf{q}_{2}}\left\{\frac{\partial}{\partial \rho_{\mathbf{q}_{1}}}+\rho_{-\mathbf{q}_{1}} w\left(\mathbf{q}_{1}\right)\right\}\left\{\frac{\partial}{\partial \rho_{\mathbf{q}_{2}}}+\rho_{-\mathbf{q}_{2}} w\left(\mathbf{q}_{2}\right)\right\} .
\end{gathered}
$$


In order to eliminate the collective term in the $\widehat{\mathcal{H}}_{W}(a \mid \rho)$ we introduce one more collective variable $\mathcal{N}$ which corresponds to the operator of the complete number of particles $\widehat{N}$ by the delta-function $\delta(\mathcal{N}-\widehat{N})$. The simplest choice of the function $w(\mathbf{q})$ from the condition

$$
1-2 \mathcal{N} w(\mathbf{q})=0
$$

leads to the following representation of the partition function:

$$
\begin{aligned}
Z= & \operatorname{Sp}_{\mathrm{a}} \int(\mathrm{d} \rho) \mathrm{d} \mathcal{N} \delta(\mathcal{N}-\widehat{N}) \widehat{J}(\rho, \widehat{\rho}) \exp \left\{-\beta\left[\widehat{H}(a)+\widehat{\mathcal{H}}(\rho)+\widehat{H}_{\text {int }}(a \mid \rho)\right]\right\} \\
\widehat{\mathcal{H}}(\rho)= & \frac{1}{2} \sum_{\mathbf{q} \in C_{\mathbf{q}}}\left\{\left[\frac{V_{\mathbf{q}}}{V}+\frac{\varepsilon_{\mathbf{q}}}{2 \mathcal{N}}\right] \rho_{\mathbf{q}} \rho_{-\mathbf{q}}-2 \mathcal{N} \varepsilon_{\mathbf{q}} \frac{\partial^{2}}{\partial \rho_{\mathbf{q}} \partial \rho_{-\mathbf{q}}}\right\} \\
& +\frac{\hbar^{2}}{2 m} \sum_{\mathbf{q}_{1}, \mathbf{q}_{2} \in C_{\mathbf{q}}}\left(\mathbf{q}_{1}, \mathbf{q}_{2}\right) \rho_{\mathbf{q}_{1}+\mathbf{q}_{2}}\left\{\frac{\partial}{\partial \rho_{\mathbf{q}_{1}}}+\frac{\rho_{-\mathbf{q}_{1}}}{2 \mathcal{N}}\right\}\left\{\frac{\partial}{\partial \rho_{\mathbf{q}_{2}}}+\frac{\rho_{-\mathbf{q}_{2}}}{2 \mathcal{N}}\right\} \\
\widehat{H}_{\text {int }}(a \mid \rho)= & \frac{\hbar^{2}}{m} \sum_{\mathbf{q} \in C_{\mathbf{q}}}\left(\frac{\rho_{-\mathbf{q}}}{2 \mathcal{N}}+\frac{\partial}{\partial \rho_{\mathbf{q}}}\right) \widehat{f}_{\mathbf{q}} \\
\widehat{H}(a) \equiv & \widehat{\mathcal{H}}_{0}+\widehat{V}_{s} ; \quad \widehat{\mathcal{H}}_{0}=\sum_{\mathbf{k}, s} \epsilon_{\mathbf{k}} a_{\mathbf{k}, s}^{+} a_{\mathbf{k}, s}
\end{aligned}
$$

where

$$
\epsilon_{\mathbf{k}}=\varepsilon_{\mathbf{k}}-\frac{1}{2 V} \sum_{\mathbf{q} \in C_{\mathbf{q}}}\left[V_{\mathbf{q}}+\left(\frac{\mathcal{N}}{V}\right)^{-1} \varepsilon_{\mathbf{q}}\right]
$$

is renormalized one-particle energy. The symbol $\mathrm{Sp}_{\mathrm{a}}$ denominates the calculation of the operation of the trace over the individual variables.

Let us introduce, instead of variables $\rho_{\mathbf{q}}$, the Bose operators of creation and destruction of plasmons $b_{\mathbf{q}}^{+}, b_{\mathbf{q}}$ using the rule:

$$
\begin{aligned}
& b_{\mathbf{q}}=\frac{1}{\sqrt{2}}\left\{\alpha_{\mathbf{q}} \tilde{\rho}_{\mathbf{q}}+\frac{1}{\alpha_{\mathbf{q}}} \frac{\partial}{\partial \tilde{\rho}_{-\mathbf{q}}}\right\}, \quad b_{-\mathbf{q}}^{+}=\frac{1}{\sqrt{2}}\left\{\alpha_{\mathbf{q}} \tilde{\rho}_{\mathbf{q}}-\frac{1}{\alpha_{\mathbf{q}}} \frac{\partial}{\partial \tilde{\rho}_{-\mathbf{q}}}\right\} ; \\
& \tilde{\rho}_{\mathbf{q}} \equiv \mathcal{N}^{-\frac{1}{2}} \rho_{\mathbf{q}} ; \quad \alpha_{\mathbf{q}}=\left\{\frac{\hbar \omega_{\mathbf{q}}}{2 \varepsilon_{\mathbf{q}}}\right\}^{\frac{1}{2}} ; \quad \hbar \omega_{\mathbf{q}}=\left\{2 \frac{V_{\mathbf{q}}}{V} \mathcal{N} \varepsilon_{\mathbf{q}}+\varepsilon_{\mathbf{q}}^{2}\right\}^{\frac{1}{2}} .
\end{aligned}
$$

The transition from the variables $\rho_{\mathbf{q}}$ to the operators $b_{\mathbf{q}}^{+}, b_{\mathbf{q}}$ is realized by means of the transition operator

$$
\widehat{J}\left(\widehat{\rho}^{(b)}, \rho\right)=\prod_{C_{\mathbf{q}}} \delta\left(\widehat{\rho}^{(b)}, \rho\right), \quad \widehat{\rho}_{\mathbf{q}}^{(b)}=(2 \mathcal{N})^{-\frac{1}{2}} \alpha_{\mathbf{q}}^{-1}\left\{b_{\mathbf{q}}+b_{-\mathbf{q}}^{+}\right\} .
$$

According to formula (5)

$$
\begin{aligned}
& \exp \{-\beta {\left.\left[\widehat{H}(a)+\widehat{\mathcal{H}}(\rho)+\widehat{H}_{\text {int }}(a \mid \rho)\right]\right\} } \\
&=\operatorname{Sp}_{b}\left\{\widehat{J}\left(\widehat{\rho}^{(b)}, \rho\right) \exp \left[-\beta\left(\widehat{H}(a)+\widehat{\mathcal{H}}_{\mathrm{p}}+\widehat{H}_{\mathrm{ep}}\right)\right]\right\}\left\{\operatorname{Sp}_{b} \widehat{J}\left(\widehat{\rho}^{(b)}, \rho\right)\right\}^{-1}
\end{aligned}
$$


The operators $\widehat{\mathcal{H}}_{\mathrm{p}}$ and $\widehat{H}_{\text {ep }}$ can be taken from the operators $\widehat{\mathcal{H}}(\rho)$ and $\widehat{H}_{\text {int }}(a \mid \rho)$ due to the transition from $\tilde{\rho}_{\mathbf{q}}, \partial / \partial \tilde{\rho}_{\mathbf{q}}$ to $b_{\mathbf{q}}^{+}$and $b_{\mathbf{q}}$ correspondingly to the expressions (23):

$$
\begin{aligned}
\widehat{\mathcal{H}}_{\mathrm{p}} & =\widehat{H}_{\mathrm{p}}+\widehat{H}_{\mathrm{pp}} ; \quad \widehat{H}_{\mathrm{p}}=\sum_{\mathbf{q} \in C_{\mathbf{q}}} \hbar \omega_{\mathbf{q}}-\left(b_{\mathbf{q}}^{+} b_{\mathbf{q}}+\frac{1}{2}\right) \\
\widehat{H}_{\mathrm{pp}} & =-\frac{\hbar^{2}}{16 m \sqrt{2 \mathcal{N}}} \sum_{\mathbf{q}_{1}, \mathbf{q}_{2} \in C_{\mathbf{q}}}\left(\mathbf{q}_{1}, \mathbf{q}_{2}\right) \alpha_{\mathbf{q}_{1}}^{-1} \alpha_{\mathbf{q}_{2}}^{-1} \alpha_{\mathbf{q}_{1}+\mathbf{q}_{2}}^{-1}\left(b_{\mathbf{q}_{1}+\mathbf{q}_{2}}^{+}+b_{-\mathbf{q}_{1}-\mathbf{q}_{2}}\right) \widehat{B}_{\mathbf{q}_{1}} \widehat{B}_{\mathbf{q}_{2}} \\
\widehat{H}_{\mathrm{ep}} & =\frac{\hbar^{2}}{2 m \sqrt{2 \mathcal{N}}} \sum_{\mathbf{q} \in C_{\mathbf{q}}} \alpha_{\mathbf{q}}^{-1} \widehat{f}_{\mathbf{q}} \widehat{B}_{\mathbf{q}} \\
\widehat{B}_{\mathbf{q}} & =\left[\frac{\hbar \omega_{\mathbf{q}}}{\varepsilon_{\mathbf{q}}}+1\right] b_{\mathbf{q}}-\left[\frac{\hbar \omega_{\mathbf{q}}}{\varepsilon_{\mathbf{q}}}-1\right] b_{-\mathbf{q}}^{+} .
\end{aligned}
$$

Let us insert the expression (25) into formula (22) and integrate over the variables $\rho_{\mathbf{q}}$ using the rule

$$
\int(\mathrm{d} \rho) \widehat{J}\left(\widehat{\rho}, \rho^{(a)}\right) \widehat{J}\left(\widehat{\rho}^{(b)}-\rho\right)\left\{\operatorname{Sp}_{b} \widehat{J}\left(\widehat{\rho}^{(b)}, \rho\right)\right\}^{-1}=\widehat{J}(b, a)=\prod_{\mathbf{q} \in C_{\mathbf{q}}} \delta\left(\widehat{\rho}_{\mathbf{q}}^{(b)}-\widehat{\rho}_{\mathbf{q}}^{(a)}\right),
$$

where $\widehat{\rho}_{\mathbf{q}}^{(a)} \equiv \widehat{\rho}_{\mathbf{q}}=\sum_{\mathbf{k}, s} a_{\mathbf{k}+\mathbf{q}, s}^{+} a_{\mathbf{k}, s}$. As a result of this procedure we obtain the partition function in the following form:

$$
Z=\operatorname{Sp}\left\{\int \mathrm{d} \mathcal{N} \delta(\mathcal{N}-\widehat{N}) \widehat{J}(b, a) \exp \left[-\beta\left(\widehat{H}(a)+\widehat{\mathcal{H}}_{\mathrm{p}}+\widehat{H}_{\mathrm{ep}}\right)\right]\right\}
$$

Formally, $\widehat{H}(a)+\widehat{\mathcal{H}}_{\mathrm{p}}+\widehat{H}_{\mathrm{ep}}$ describes two different subsystems of the objects: electrons and plasmons. The operator $\widehat{J}(b, a)$ represents the subsidiary conditions which are necessary for this description.

The following calculation of the partition function is done within the framework of the perturbation theory. To this end, let us use the interaction representation in the statistical operator

$$
\exp \left[-\beta\left(\widehat{H}(a)+\widehat{\mathcal{H}}_{\mathrm{p}}+\widehat{H}_{\mathrm{ep}}\right)\right]=\exp \left\{-\beta\left(\widehat{\mathcal{H}}_{0}+\widehat{H}_{p}\right)\right\} T \widehat{S}
$$

where $T$ is the chronological ordering symbol and $\widehat{S}$-matrix is determined by the operators of interactions:

$$
\widehat{S}=\exp \left\{-\int_{0}^{\beta} \mathrm{d} \beta^{\prime}\left[\widehat{V}_{s}\left(\beta^{\prime}\right)+\widehat{H}_{\mathrm{pp}}\left(\beta^{\prime}\right)+\widehat{H}_{\mathrm{ep}}\left(\beta^{\prime}\right)\right]\right\} .
$$

Moving the operator $\exp \left\{-\beta\left[\widehat{\mathcal{H}}_{0}+\widehat{H}_{\mathrm{p}}\right]\right\}$ through the transition operator $\widehat{J}(b, a)$ we can translate the latter in the interaction representation:

$Z=\operatorname{Sp}_{a, b}\left\{\int \mathrm{d} \mathcal{N} \delta(\mathcal{N}-\widehat{N}) \exp \left[-\beta\left(\widehat{\mathcal{H}}_{0}+\widehat{H}_{\mathrm{p}}\right)\right] T\left[\prod_{C_{\mathbf{q}}} \delta\left(\widehat{\rho}_{\mathbf{q}}^{(b)}(\beta)-\widehat{\rho}_{\mathbf{q}}^{(a)}(\beta)\right) \widehat{S}\right]\right\}$ 


$$
\widehat{\rho}_{\mathbf{q}}^{(a)}(\beta) \equiv \sum_{\mathbf{k}, s} a_{\mathbf{k}+\mathbf{q}, s}^{+}(\beta) a_{\mathbf{k}, s}(\beta) ; \quad \widehat{\rho}_{\mathbf{q}}^{(b)}(\beta) \equiv 2^{-\frac{1}{2}} N^{\frac{1}{2}} \alpha_{\mathbf{q}}^{-1}\left[b_{\mathbf{q}}(\beta)+b_{-\mathbf{q}}^{+}(\beta)\right] .
$$

Let us transit to the so-called "frequency" representation $[26,27]$ in the following calculation of the perturbation theory diagrams introducing the combinations of the operators $a_{\mathbf{k}, s}\left(\beta^{\prime}\right)$ and $b_{\mathbf{q}}\left(\beta^{\prime}\right)$ :

$$
a_{\mathbf{k}, s}\left(\nu^{*}\right)=\int_{0}^{\beta} a_{\mathbf{k}, s}\left(\beta^{\prime}\right) \Psi_{\nu^{*}}\left(\beta^{\prime}\right) \mathrm{d} \beta^{\prime}, \quad b_{\mathbf{q}}(\nu)=\int_{0}^{\beta} b_{\mathbf{q}}\left(\beta^{\prime}\right) \Psi_{\nu}\left(\beta^{\prime}\right) \mathrm{d} \beta^{\prime},
$$

where $\Psi_{\nu}\left(\beta^{\prime}\right)=\beta^{-\frac{1}{2}} \exp \left(\mathrm{i} \nu \beta^{\prime}\right), \nu^{*}=(2 n+1) \pi \beta^{-1}, \nu=2 \pi n \beta^{-1}, n=0 ; \pm 1 ; \pm 2 ; \ldots$ In the new representation

$$
\begin{aligned}
\widehat{S} \equiv & \widehat{S}(\nu)=\exp \left\{-\widehat{V}_{s}(\nu)-\widehat{H}_{\mathrm{pp}}(\nu)-\widehat{H}_{\mathrm{ep}}(\nu)\right\} \\
\widehat{V}_{s}(\nu)= & \frac{1}{2 \beta V} \sum_{\mathbf{q} \in C_{\mathbf{q}}} \sum_{\nu} V_{\mathbf{q}} \sum_{\mathbf{k}_{1}, \mathbf{k}_{2}} \sum_{s_{1}, s_{2}} \sum_{\nu_{1}^{*}, \nu_{2}^{*}} a_{\mathbf{k}_{1}+\mathbf{q}, s_{1}}^{+}\left(\nu_{1}^{*}+\nu\right) \\
& \times a_{\mathbf{k}_{2}-\mathbf{q}, s_{2}}^{+}\left(\nu_{2}^{*}-\nu\right) a_{\mathbf{k}_{2}, s_{2}}\left(\nu_{2}^{*}\right) a_{\mathbf{k}_{1}, s_{1}}\left(\nu_{1}^{*}\right) ; \\
\widehat{H}_{\mathrm{ep}}(\nu)= & \frac{\hbar^{2}}{2 m \sqrt{2 \mathcal{N} \beta}} \sum_{\mathbf{q} \in C_{\mathbf{q}}} \sum_{\nu} \alpha_{\mathbf{q}}^{-1} \widehat{f}_{\mathbf{q}, \nu} \widehat{B}_{\mathbf{q}, \nu} ; \\
\widehat{B}_{\mathbf{q}, \nu}= & \left\{\left(\frac{\hbar \omega_{\mathbf{q}}}{\varepsilon_{\mathbf{q}}}+1\right) b_{\mathbf{q}, \nu}-\left(\frac{\hbar \omega_{\mathbf{q}}}{\varepsilon_{\mathbf{q}}}-1\right) b_{-\mathbf{q},-\nu}^{+}\right\} ; \\
\widehat{f}_{\mathbf{q}, \nu}= & \sum_{\mathbf{k}, s} \sum_{\nu^{*}}(\mathbf{k q}) a_{\mathbf{k}+\mathbf{q}, s}^{+}\left(\nu^{*}+\nu\right) a_{\mathbf{k}, s}\left(\nu^{*}\right) ; \\
\widehat{H}_{\mathrm{pp}}(\nu)= & \frac{\hbar^{2}}{4 m \sqrt{2 \mathcal{N} \beta}} \sum_{\mathbf{q}_{1}, \mathbf{q}_{2} \in C_{\mathbf{q}}}\left(\mathbf{q}_{1}, \mathbf{q}_{2}\right) \sum_{\nu_{1}, \nu_{2}} \alpha_{\mathbf{q}_{1}+\mathbf{q}_{2}}^{-1}\left(b_{x_{1}+x_{2}}+b_{-x_{1}-x_{2}}^{+}\right) \\
& \times \prod_{j=1,2}\left\{\alpha_{\mathbf{q}_{j}}\left(b_{x_{j}}-b_{-x_{j}}^{+}\right)+\frac{1}{2 \alpha_{\mathbf{q}_{j}}}\left(b_{-x_{j}}+b_{x_{j}}^{+}\right)\right\}
\end{aligned}
$$

where $b_{x} \equiv b_{\mathbf{q}, \nu}$. Since

$$
\begin{aligned}
& \widehat{\rho}_{\mathbf{q}}^{(a)}(\beta)=\beta^{-1} \sum_{\nu} \widehat{\rho}_{\mathbf{q}, \nu}, \quad \widehat{\rho}_{\mathbf{q}, \nu} \equiv \rho_{x}=\sum_{\mathbf{k}, s} \sum_{\nu^{*}} a_{\mathbf{k}+\mathbf{q}, s}^{+}\left(\nu^{*}+\nu\right) a_{\mathbf{k}, s}\left(\nu^{*}\right), \\
& \widehat{\rho}_{\mathbf{q}}^{(b)}(\beta)=\sqrt{\frac{\mathcal{N}}{2 \beta}} \alpha_{\mathbf{q}}^{-1} \sum_{\nu}\left(b_{\mathbf{q}, \nu}+b_{-\mathbf{q},-\nu}^{+}\right),
\end{aligned}
$$

then using the integral representation of $\delta$-function in the expression (31) we can obtain such a working formula to calculate the partition function:

$$
Z=\int_{-\infty}^{+\infty} \mathrm{d} \mathcal{N} Z_{0} \int_{-\infty}^{+\infty} \mathrm{d} \omega_{0} \exp \left(2 \pi \mathrm{i} \omega_{0} \mathcal{N}\right) \xi\left(\omega_{0}\right)
$$




$$
\begin{aligned}
& \times\left\langleT \left\{\int(\mathrm{d} \varphi) \exp \left(\mathrm{i} \pi \sum_{\nu} \sum_{\mathbf{q} \in C_{\mathbf{q}}} \varphi_{\mathbf{q}}\left[\sqrt{\frac{\mathcal{N}}{2 \beta}} \alpha_{\mathbf{q}}^{-1}\left(b_{x}+b_{-x}^{+}\right)-\frac{1}{\beta} \widehat{\rho}_{x}\right]\right)\right.\right. \\
& \left.\left.\times \exp \left[-\widehat{V}_{s}(\nu)-\widehat{H}_{\mathrm{pp}}(\nu)-\widehat{H}_{\mathrm{ep}}(\nu)\right]\right\}\right\rangle_{0} ; \quad(\mathrm{d} \varphi) \equiv \prod_{C_{\mathbf{q}}} \mathrm{d} \varphi_{\mathbf{q}} .
\end{aligned}
$$

The statistical averaging is done over the states of free electrons and free plasmons,

$$
Z_{0}=\operatorname{Sp}\left\{\exp \left[-\beta\left(\widehat{\mathcal{H}}_{0}+\widehat{H}_{\mathrm{p}}\right)\right]\right\}=\exp \left(-\beta \Omega_{0}\right)
$$

is partition function of free electrons and free plasmons and $\Omega_{0}$ is the grand thermodynamic potential

$$
\Omega_{0}=-\frac{1}{\beta} \sum_{\mathbf{k}, s} \ln \left\{1+\mathrm{e}^{-\beta\left(\varepsilon_{\mathbf{k}}-\mu\right)}\right\}+\frac{1}{\beta} \sum_{\mathbf{q} \in C_{\mathbf{q}}} \ln \left\{1-\mathrm{e}^{-\beta \hbar \omega_{\mathbf{q}}}\right\}+\frac{1}{2} \sum_{\mathbf{q} \in C_{\mathbf{q}}} \hbar \omega_{\mathbf{q}} .
$$

The factor $\xi\left(\omega_{0}\right)$ is determined by the following correlation:

$$
\begin{aligned}
\xi\left(\omega_{0}\right) & =\left\{\operatorname{Sp}_{\mathrm{a}} \exp \left[-\beta\left(\widehat{\mathcal{H}}_{0}-\mu \widehat{N}\right)\right]\right\}^{-1} \operatorname{Sp}_{\mathrm{a}} \exp \left\{-\beta\left[\widehat{\mathcal{H}}_{0}-\mu \widehat{N}\right]-2 \pi \mathrm{i} \omega_{0} \widehat{N}\right\} \\
& =\prod_{\mathbf{k}, s}\left\{1-n_{\mathbf{k}, s}+n_{\mathbf{k}, s} \exp \left(-2 \pi \mathrm{i} \omega_{0}\right)\right\}
\end{aligned}
$$

where $n_{\mathbf{k}, s}=\left\{1+\exp \left[\beta\left(\varepsilon_{\mathbf{k}}-\mu\right)\right]\right\}^{-1}$ is the distribution Fermi in the ideal system of electrons. From the formula (38) we can see that in the limit of low temperatures at the full degeneration $(\beta \rightarrow \infty)$ the $\xi\left(\omega_{0}\right) \rightarrow \exp \left(-2 \pi \mathrm{i} \omega_{0} N\right)$ where $N$ is the number of electrons in the system. In the general case

$$
\xi\left(\omega_{0}\right)=\exp \left\{-2 \pi \mathrm{i} \omega_{0} N+\sum_{n \geqslant 2}(-2 \pi \mathrm{i})^{n} \frac{\omega_{0}^{n}}{n !} \beta^{1-n} \mu_{n}^{0}(0)\right\},
$$

where $\mu_{n}^{0}(0)$ is the statical long-wave limit of the $n$-particle dynamic correlation functions of the ideal system [26]

$$
\mu_{n}^{0}\left(x_{1}, \ldots, x_{n}\right)=\frac{1}{\beta}\left\langle\widehat{\rho}_{x_{1}} \widehat{\rho}_{x_{2}} \cdots \widehat{\rho}_{x_{n}}\right\rangle_{0} .
$$

For $\mu_{n}^{0}(0)$ we have the following expression:

$$
\mu_{n}^{0}(0)=\sum_{\mathbf{k}, s} \frac{d^{n-1}}{d \mu^{n-1}} n_{\mathbf{k}, s}=(-1)^{n} \frac{V}{\pi^{2}} \frac{\mu^{\frac{5}{2}-n}}{2^{n-1}}\left(\frac{2 m}{\hbar^{2}}\right)^{\frac{3}{2}}\left\{R_{n}-\frac{\pi^{2}}{24}(\beta \mu)^{-2}(2 n-3) ! !+\cdots\right\},
$$

where $R_{2}=1, R_{3}=1$ also at $n \geqslant 4$ we have $R_{n}=-(2 n-7) !$ !.

The identity

$$
\left\{2 \pi \mathrm{i} \omega_{0}+\frac{\partial}{\partial N}\right\} \exp \left(-2 \pi \mathrm{i} \omega_{0} N\right)=0
$$


makes it possible to integrate over variables $\omega_{0}$ and $\mathcal{N}$ and brings the calculation of partition function to the following form:

$$
\begin{aligned}
Z= & \widehat{\Lambda}(N) Z_{0}\left\langleT \left\{\int(\mathrm{d} \varphi) \exp \left(\mathrm{i} \pi \sum_{\nu} \sum_{\mathbf{q} \in C_{\mathbf{q}}} \varphi_{\mathbf{q}}\left[\sqrt{\frac{\mathcal{N}}{2 \beta}} \alpha_{\mathbf{q}}^{-1}\left(b_{x}+b_{-x}^{+}\right)-\frac{1}{\beta} \widehat{\rho}_{x}\right]\right)\right.\right. \\
& \left.\left.\times \exp \left[-\widehat{V}_{s}(\nu)-\widehat{H}_{\mathrm{pp}}(\nu)-\widehat{H}_{\mathrm{ep}}(\nu)\right]\right\}\right\rangle_{0}
\end{aligned}
$$

Hence, in the $Z_{0}$ as well as in the brackets $\langle\cdots\rangle_{0}$ it is necessary to supply everywhere the number of particles $N$ in place of variables $\mathcal{N}$. The operator $\widehat{\Lambda}(N)$ appears at the expense of the terms $n \geqslant 2$ in the formula (39) and takes into account the temperature corrections:

$$
\widehat{\Lambda}(N)=\exp \left\{\sum_{n \geqslant 2}(n !)^{-1} \beta^{1-n} \mu_{n}^{0}(0) \frac{\partial^{n}}{\partial N^{n}}\right\} .
$$

It is advisable to write the formula (43) to the equivalent form extracting the partition function $Z_{\mathrm{ep}}$ of the model system which consists of interaction subsystems of electrons and plasmons, but without taking into account the subsidiary conditions, namely

$$
\begin{aligned}
Z & =\widehat{\Lambda}(N) Z_{\mathrm{ep}}\langle\widehat{J}(b, a)\rangle_{\mathrm{ep}} ; \quad Z_{\mathrm{ep}}=Z_{0}\langle T \widehat{S}(\nu)\rangle_{0} ; \\
\langle\widehat{J}(b, a)\rangle_{\mathrm{ep}} & =\left\langle\int(\mathrm{d} \varphi) \exp \left\{\mathrm{i} \pi \sum_{\mathbf{q} \in C_{\mathbf{q}}} \sum_{\nu} \varphi_{\mathbf{q}}\left[N^{\frac{1}{2}}(2 \beta)^{-\frac{1}{2}} \alpha_{\mathbf{q}}^{-1}\left(b_{x}+b_{-x}^{+}\right)-\frac{1}{\beta} \widehat{\rho}_{x}\right]\right\}\right\rangle_{\mathrm{ep}} \\
\langle\widehat{A}\rangle_{\mathrm{ep}} & \equiv\langle T\{\widehat{A} \widehat{S}(\nu)\}\rangle_{0}\langle T \widehat{S}(\nu)\rangle_{0}^{-1}
\end{aligned}
$$

\section{Equivalence of the electron liquid and electron-plasmon models in the random phase approximation}

In order to determine the correspondence of our approach to the traditional approach of the perturbation theory over the powers of Coulomb potential let us consider the simplest variant where $q_{0} \rightarrow \infty$, the short-range interaction $\widehat{\tilde{V}}_{\text {ee }}(\nu)$ is absent as well as the system is found in the ground state $(T=0 \mathrm{~K})$.

First, let us calculate $Z_{\text {ep }}$ in the RPA. It allows us to neglect the operator $\widehat{H}_{\mathrm{pp}}(\nu)$ and $\widehat{S}$-matrix can be transformed into the form $\exp \left(-\widehat{H}_{\mathrm{ep}}(\nu)\right)$. Unlike the zero ones, contributions arise only in the pair orders of the perturbation theory since the operator $\widehat{H}_{\mathrm{ep}}(\nu)$ is linear relatively to the plasmon operators $b_{\mathbf{q}, \nu}, b_{\mathbf{q}, \nu}^{+}$. The other important specific characteristic is the absence of divergent diagrams in any order of the perturbation theory. It is caused by the structure of the operator $\widehat{H}_{\mathrm{ep}}(\nu)$. For example, 
let us consider the contribution in the thermodynamic potential single diagram of the second order of the perturbation theory:

$$
\begin{aligned}
\Delta \Omega_{2} & =-\frac{1}{2 \beta}\left\langle T \widehat{H}_{\mathrm{ep}}^{2}(\nu)\right\rangle_{0} \\
& =-\sum_{\mathbf{q}, \nu}\left(\frac{\hbar^{2}}{2 m}\right)^{2}\left(4 N \beta^{2} \alpha_{\mathbf{q}}^{2}\right)^{-1}\left\langle T\left\{\widehat{f}_{x} \widehat{f}_{-x}\right\}\right\rangle_{0}\left\langle T\left\{\widehat{B}_{x} \widehat{B}_{-x}\right\}\right\rangle_{0} .
\end{aligned}
$$

Here, the average values are determinated per spectrum representation of the Green's functions of the electrons and plasmons [27]

$$
\begin{aligned}
-\left\langle T\left\{a_{\mathbf{k}_{1}, s_{1}}\left(\nu_{1}^{*}\right) a_{\mathbf{k}_{2}, s_{2}}^{+}\left(\nu_{2}^{*}\right)\right\}\right\rangle_{0} & =G_{\mathbf{k}_{1}, s_{1}}^{e}\left(\nu_{1}^{*}\right) \delta_{s_{1}, s_{2}} \delta_{\mathbf{k}_{1}, \mathbf{k}_{2}} \delta_{\nu_{1}^{*}, \nu_{2}^{*}} ; \\
G_{\mathbf{k}, s}^{e}\left(\nu^{*}\right) & =\left\{\mathrm{i} \nu^{*}-\varepsilon_{\mathbf{k}}+\mu\right\}^{-1} ; \\
-\left\langle T\left\{b_{x} b_{x}^{+}\right\}\right\rangle_{0} & =G_{\mathbf{q}}^{p}(\nu)=\left\{\mathrm{i} \nu-\hbar \omega_{\mathbf{q}}\right\}^{-1} .
\end{aligned}
$$

According to the expressions (47),

$$
\begin{aligned}
\left\langle T\left\{\widehat{B}_{x} \widehat{B}_{-x}\right\}\right\rangle_{0} & =-2 \hbar \omega_{\mathbf{q}}\left\{\left(\frac{\hbar \omega_{\mathbf{q}}}{\varepsilon_{\mathbf{q}}}\right)^{2}-1\right\}\left\{\nu^{2}+\left(\hbar \omega_{\mathbf{q}}\right)^{2}\right\}^{-1} \\
\left\langle T\left\{\widehat{f}_{x} \widehat{f}_{-x}\right\}\right\rangle_{0} & =\sum_{\mathbf{k}, s} \sum_{\nu^{*}}(\mathbf{k}, \mathbf{q})(\mathbf{k}+\mathbf{q}, \mathbf{q}) G_{\mathbf{k}, s}^{e}\left(\nu^{*}\right) G_{\mathbf{k}+\mathbf{q}, s}^{e}\left(\nu^{*}+\nu\right) .
\end{aligned}
$$

Performing the elementary transformation and using the summation rule over the frequencies

$$
\beta^{-1} \sum_{\nu^{*}} G_{\mathbf{k}, s}^{e}\left(\nu^{*}\right)=n_{\mathbf{k}, s}
$$

we find

$$
\beta^{-1}\left(\frac{\hbar^{2}}{2 m}\right)^{2}\left\langle T\left\{\widehat{f}_{x} \widehat{f}_{-x}\right\}\right\rangle_{0}=-\frac{1}{2}\left\{N \varepsilon_{\mathbf{q}}-\frac{1}{2}\left(\nu^{2}+\varepsilon_{\mathbf{q}}^{2}\right) \mu_{2}^{0}(x,-x)\right\},
$$

where

$$
\begin{aligned}
\mu_{2}^{0}(x,-x) & =\beta^{-1}\left\langle T\left\{\widehat{\rho}_{x} \widehat{\rho}_{-x}\right\}\right\rangle_{0}=-\beta^{-1} \sum_{\mathbf{k}, s} \sum_{\nu^{*}} G_{\mathbf{k}, s}^{e}\left(\nu^{*}\right) G_{\mathbf{k}+\mathbf{q}, s}^{e}\left(\nu^{*}+\nu\right) \\
& =3 N\left(2 \varepsilon_{\mathrm{F}}\right)^{-1} I_{2,0}(q, u)
\end{aligned}
$$

- is the spectrum representation of the two-particle correlation function of the electron system without interaction in the variables "wave vector - frequency Matsubara". Non-dimensional factor $I_{2,0}(q, u)$ at absolute zero temperature can be represented by this well-known expression [8]:

$$
\begin{aligned}
I_{2,0}(q, u)= & \frac{1}{2}\left\{1+\frac{1}{2 q}\left(1+u^{2}-\frac{q^{2}}{4}\right) \sum_{\sigma= \pm 1} \sigma \ln \left[\left(1+\sigma \frac{q}{2}\right)^{2}+u^{2}\right]\right. \\
& \left.-u \sum_{\sigma= \pm 1} \operatorname{arctg}\left[\frac{1}{u}\left(1+\sigma \frac{q}{2}\right)\right]\right\} ; \\
q \equiv & |\mathbf{q}| k_{F}^{-1} ; \quad u \equiv \nu\left(2 \varepsilon_{\mathrm{F}} q\right)^{-1} ; \quad \varepsilon_{\mathrm{F}}=\hbar^{2} k_{\mathrm{F}}^{2}(2 m)^{-1} .
\end{aligned}
$$


Let us show the ground state energy of the model in the traditional form

$$
E=E_{\mathrm{id}}+E_{\mathrm{HF}}+E_{\mathrm{corr}}
$$

where $E_{\text {id }}$ is the energy of the ideal electron system, $E_{\mathrm{HF}}$ is contribution in the Hartree-Fock approximation, $E_{\text {corr }}$ is a contribution of the correlation caused by interactions (the so-called, correlation energy).

In the zero approximation of operator $\widehat{H}_{\mathrm{ep}}(\nu)$ we can have the following expression for the correlation energy

$$
E_{\text {corr }}^{(0)}=\frac{1}{2} \sum_{\mathbf{q}}\left\{\hbar \omega_{\mathbf{q}}-\varepsilon_{\mathbf{q}}-\frac{N}{V} V_{\mathbf{q}}\left[1-\frac{1}{N} \sum_{\mathbf{k}, s} n_{\mathbf{k}, s} n_{\mathbf{k}+\mathbf{q}, s}\right]\right\}
$$

Summing over the frequency in the terms of formula (46) which does not have $\mu_{2}^{0}(x,-x)$ in the second order of the perturbation theory over operator $\widehat{H}_{\mathrm{ep}}(\nu)$ for correlation energy, we obtain

$$
\begin{aligned}
E_{\mathrm{corr}}^{(2)}= & \frac{1}{2} \sum_{\mathbf{q}}\left\{\hbar \omega_{\mathbf{q}}-\varepsilon_{\mathbf{q}}-\frac{N}{V} V_{\mathbf{q}} \frac{\varepsilon_{\mathbf{q}}}{\hbar \omega_{\mathbf{q}}}\right\} \\
& -\frac{1}{2 \beta V} \sum_{\mathbf{q}, \nu} V_{\mathbf{q}} \mu_{2}^{0}(x,-x)\left\{\left(\hbar \omega_{\mathbf{q}}\right)^{2}-\varepsilon_{\mathbf{q}}^{2}\right\}\left\{\nu^{2}+\left(\hbar \omega_{\mathbf{q}}\right)^{2}\right\}^{-1}
\end{aligned}
$$

The summation of infinite series of non-divergent ring diagrams constructed on the operator $\widehat{H}_{\mathrm{ep}}(\nu)$ brings to the following representation of the ground state of the energy of the model:

$$
E=E_{\mathrm{id}}+\frac{1}{2} \sum_{\mathbf{q}}\left\{\hbar \omega_{\mathbf{q}}-\varepsilon_{\mathbf{q}}-\frac{N}{V} V_{\mathbf{q}}\right\}+\frac{1}{2 \beta} \sum_{\nu} \sum_{\mathbf{q}} \ln \left\{1-\left\langle T \widehat{H}_{\mathrm{ep}}^{2}(\nu)\right\rangle_{0}^{\mathbf{q}, \nu}\right\}
$$

Here, $\left\langle T \widehat{H}_{\mathrm{ep}}^{2}(\nu)\right\rangle_{0}^{\mathbf{q}, \nu}$ is $(\mathbf{q}, \nu)$-component of the average $\left\langle T \widehat{H}_{\mathrm{ep}}^{2}(\nu)\right\rangle_{0}$ :

$$
\left\langle T \widehat{H}_{\mathrm{ep}}^{2}(\nu)\right\rangle_{0}^{\mathbf{q}, \nu}=2\left\{N \varepsilon_{\mathbf{q}}-\frac{1}{2}\left(\nu^{2}+\varepsilon_{\mathbf{q}}^{2}\right) \mu_{2}^{0}(x,-x)\right\} \frac{V_{\mathbf{q}}}{V}\left\{\nu^{2}+\left(\hbar \omega_{\mathbf{q}}\right)^{2}\right\}^{-1} .
$$

Taking into account the expressions (48), (50) after elementary transformations the logarithm from the formula (56) has the following representation:

$$
\begin{aligned}
& \ln \left[1-\left\langle T \widehat{H}_{\mathrm{ep}}^{2}(\nu)\right\rangle_{0}^{\mathbf{q}, \nu}\right]=\ln \left[1+\frac{V_{\mathbf{q}}}{V} \mu_{2}^{0}(x,-x)\right]+L(x), \\
& L(x)=\ln \left\{1-\left[\left(\hbar \omega_{\mathbf{q}}\right)^{2}-\varepsilon_{\mathbf{q}}^{2}\right]\left[\left(\hbar \omega_{\mathbf{q}}\right)^{2}+\nu^{2}\right]^{-1}\right\} .
\end{aligned}
$$

Let us calculate the sum over the frequency $\nu$ from $L(x)$,

$$
\beta^{-1} \sum_{\nu} L(x)=(2 \pi)^{-1} \int_{-\infty}^{+\infty} L(x) \mathrm{d} \nu=-\left(\hbar \omega_{\mathbf{q}}-\varepsilon_{\mathbf{q}}\right)
$$


Extracting the contribution $E_{\mathrm{HF}}$ from the (56) we can obtain a familiar standard expression for correlation energy in the RPA

$$
E_{\mathrm{corr}}^{\mathrm{RPA}}=\frac{1}{2 \beta} \sum_{\mathbf{q}, \nu}\left\{\ln \left[1+\frac{V_{\mathbf{q}}}{V} \mu_{2}^{0}(x,-x)\right]-\frac{V_{\mathbf{q}}}{V} \mu_{2}^{0}(x,-x)\right\},
$$

that adequately describes the model in the weak non-ideality range $\left(r_{s} \leqslant 1\right)$. This expression is the sum of the most divergent ordinary perturbation theory diagrams which are constructed on the Coulomb potential [8]. It proves the total equivalence of the electron-plasmon model and the ordinary model with Coulomb interaction in RPA.
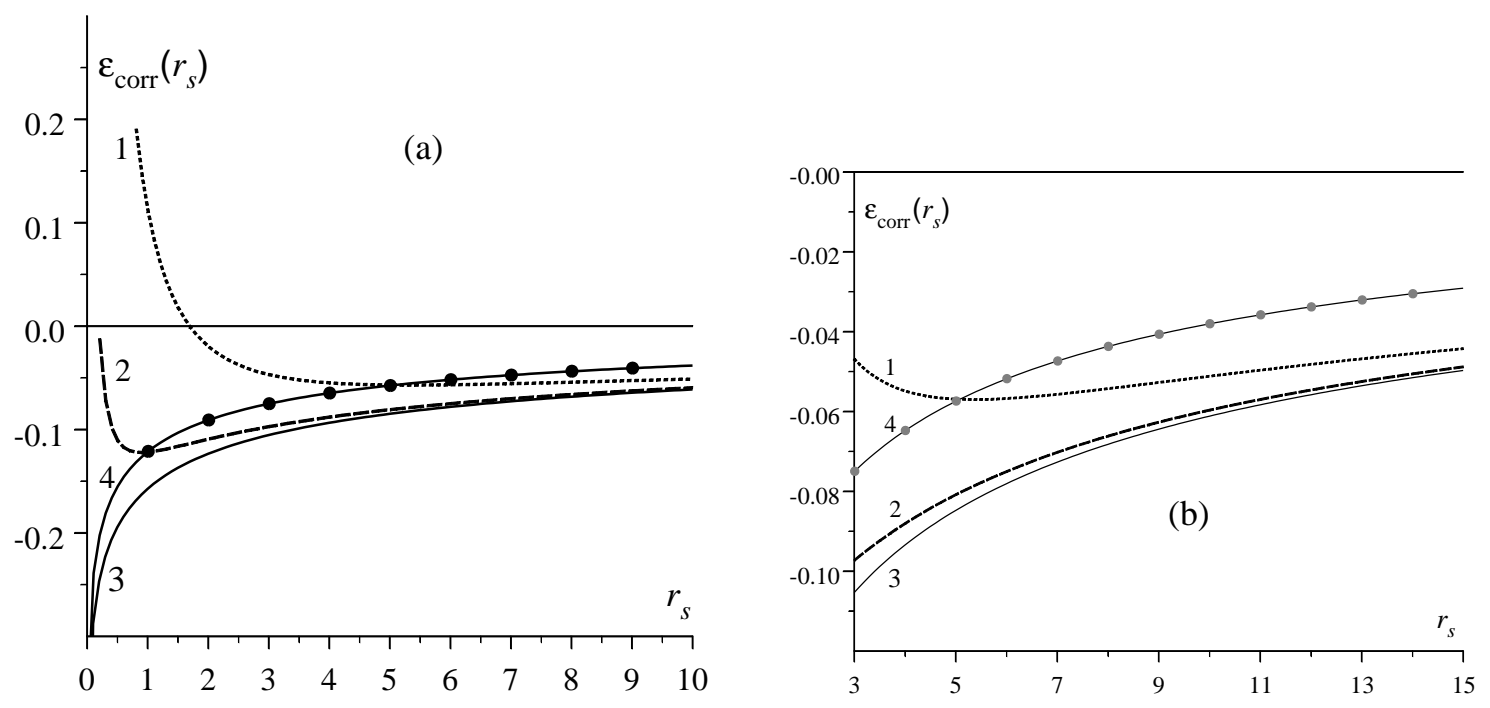

Figure 1. (a): The ground state correlation energy of electron liquid model (in Ry per electron) in different approximations: curve 1 corresponds to (60), curve 2 is the expression of (61), curve 3 is the random phase approximation, curve 4 the results of the calculation by means of the Monte-Carlo method [9].

(b): The ground state correlation energy of electron liquid model in intermediate and strong non-ideality region. Notations are similar to figure 1a.

The dependence of the ground state correlation energy of electron liquid model in the Ry per electron $\left(\varepsilon_{\text {corr }}\left(r_{s}\right)=[N R y]^{-1} E_{\text {corr }}\right)$ on the parameter non-ideality

$$
r_{s}=\left(\frac{3 V}{4 \pi N}\right)^{\frac{1}{3}} \frac{m e^{2}}{\hbar^{2}}
$$

(the Brueckner parameter) in different approximations is shown in figures 1a, $1 \mathrm{~b}$. Curve 1 corresponds to approximation (54) where

$$
\varepsilon_{\mathrm{corr}}^{(0)}\left(r_{s}\right)=\frac{A_{0}}{r_{s}}-\frac{B_{0}}{r_{s}^{3 / 4}}
$$




$$
\begin{aligned}
& A_{0}=\frac{3}{2 \pi} \eta=\frac{3}{2 \pi}\left(\frac{9 \pi}{4}\right)^{\frac{1}{3}}=0.91633 \ldots \\
& B_{0}=\frac{32}{5 \pi} \eta^{\frac{3}{4}}(3 \pi)^{-\frac{1}{4}}\left\{E\left(\frac{\pi}{2}, \frac{1}{\sqrt{2}}\right)-\frac{1}{2} F\left(\frac{\pi}{2}, \frac{1}{\sqrt{2}}\right)\right\}=0.80306 \ldots
\end{aligned}
$$

$(E(\ldots), F(\ldots)$ are the elliptic integrals $[23])$.

Approximation (55), where

$$
\varepsilon_{\text {corr }}^{(2)}\left(r_{s}\right)=\frac{B_{0}}{4 r_{s}^{\frac{3}{4}}}-\frac{32}{\pi^{3}} \int_{0}^{\infty} \mathrm{d} q q \int_{0}^{\infty} \mathrm{d} u I_{2,0}(q, u)\left\{q^{4}+4 q^{2} u^{2}+\frac{16 r_{s}}{3 \pi \eta}\right\}^{-1},
$$

corresponds to the curve 2 . Curve 3 represents the correlation energy in the RPA (see formula (54)) and curve 4 represents the calculation results of the correlation energy by means of the Monte-Carlo method [9], which is basic.

The summation infinite series (non-divergent) diagrams constructed on the powers of the operator $\widehat{H}_{\mathrm{ep}}(\nu)$ is necessary only in the weak and medium non-ideality region $\left(r_{s} \leqslant 4\right)$, which is evident in the figures. Considering the second order of the perturbation theory over the operator of the electron-plasmon interaction in the strong non-ideality region we obtain the result which coincides with the result in the RPA of the ordinary perturbation theory. Therefore, it is not necessary to do the summation of a series of diagrams over the powers of operator $\widehat{H}_{\mathrm{ep}}(\nu)$. It is an interesting and profitable characteristic of the electron-plasmon model.

Let us note that we use the approximation $q_{0} \rightarrow \infty$ only for a clearer account. In fact the result (59) does not depend on the value $q_{0}$. At the finite value $q_{0}$, in place of the formula (56), we obtain

$$
\begin{aligned}
E= & E_{\mathrm{id}}+\frac{1}{2 \beta} \sum_{\nu} \sum_{\mathbf{q} \in C_{\mathbf{q}}} \ln \left\{1-\left\langle T \widehat{H}_{\mathrm{ep}}^{2}(\nu)\right\rangle_{0}^{\mathbf{q}, \nu}\right\}+\frac{1}{2} \sum_{\mathbf{q} \in C_{\mathbf{q}}}\left[\hbar \omega_{\mathbf{q}}-\epsilon_{\mathbf{q}}-\frac{N}{V} V_{\mathbf{q}}\right] \\
& +\frac{1}{2 \beta} \sum_{\nu} \sum_{\mathbf{q} \notin C_{\mathbf{q}}} \ln \left\{1+\frac{V_{\mathbf{q}}}{V} \mu_{2}^{0}(x,-x)\right\}
\end{aligned}
$$

According to the expressions (57), (58) we obtain

$$
E=E_{\text {id }}+\frac{1}{2 \beta} \sum_{\nu} \sum_{\mathbf{q}} \ln \left\{1+\frac{V_{\mathbf{q}}}{V} \mu_{2}^{0}(x,-x)\right\}
$$

From the expression (63) we can obtain the formula (59).

Let us consider the factor $\langle\widehat{J}(a, b)\rangle$ from the (45) and its contribution to the grand thermodynamic potential in the RPA. Expanding the exponent

$$
\exp \left\{i \pi \sum_{\mathbf{q} \in C_{\mathbf{q}}} \varphi_{\mathbf{q}} \sum_{\nu}[\cdots]\right\}
$$


to the Taylor series of the powers $\varphi_{\mathbf{q}}$, one can take into account the items proportional to the $\varphi_{\mathbf{q}} \varphi_{\mathbf{q}}$ that correspond to RPA:

$$
\begin{aligned}
& \langle\widehat{J}(a, b)\rangle_{\mathrm{ep}}=\int(\mathrm{d} \varphi) \exp \left\{-\frac{\pi^{2} N}{2} \sum_{\mathbf{q}} \varphi_{\mathbf{q}} \varphi_{-\mathbf{q}}\right. \\
& \quad \times\left[\left(2 \beta \alpha_{\mathbf{q}}^{2}\right)^{-1} \sum_{\nu}\left\langle T\left(b_{x}+b_{-x}^{+}\right)\left(b_{-x}+b_{x}\right)\right\rangle_{\mathrm{ep}}+N^{-1} \beta^{-2} \sum_{\nu}\left\langle T\left(\widehat{\rho}_{x} \widehat{\rho}_{-x}\right)\right\rangle_{\mathrm{ep}}\right. \\
& \left.\left.\quad-(2 N)^{\frac{1}{2}} \beta^{-\frac{3}{2}} \alpha_{\mathbf{q}}^{-1} N^{-1} \sum_{\nu}\left\langle T\left[\left(b_{x}+b_{-x}^{+}\right) \widehat{\rho}_{-x}\right]\right\rangle_{\mathrm{ep}}\right]\right\} .
\end{aligned}
$$

The averages in the above expression are easy to calculate in the RPA. For example,

$$
\begin{aligned}
\beta^{-1} & \left\langle T\left\{\widehat{\rho}_{x} \widehat{\rho}_{-x}\right\}\right\rangle_{\mathrm{ep}}=\beta^{-1}\left\langle T\left\{\widehat{\rho}_{x} \widehat{\rho}_{-x} \mathrm{e}^{-\widehat{H}_{\mathrm{ep}}(\nu)}\right\}\right\rangle_{0}^{c}=\beta^{-1}\left\langle T\left\{\widehat{\rho}_{x} \widehat{\rho}_{-x}\right\}\right\rangle_{0} \\
& +\left(2 N \beta^{2}\right)^{-1} \alpha_{\mathbf{q}}^{-2}\left\langle T\left\{\widehat{\rho}_{x} \widehat{f}_{-x}\right\}\right\rangle_{0}\left\langle T\left\{\widehat{\rho}_{-x} \widehat{f}_{x}\right\}\right\rangle_{0}\left(\frac{\hbar^{2}}{2 m}\right)^{2}\left\{1-\left\langle T\left[\widehat{H}_{\mathrm{ep}}^{2}(\nu)\right]\right\rangle_{0}^{\mathbf{q}, \nu}\right\}^{-1} \\
& =\mu_{2}^{0}(x,-x)\left\{1+V^{-1} V_{\mathbf{q}} \mu_{2}^{0}(x,-x)\right\}^{-1} \equiv \mu_{2}^{\mathrm{RPA}}(x,-x) .
\end{aligned}
$$

It is taken into account that

$$
\hbar^{2}(2 m \beta)^{-1}\left\langle T\left\{\widehat{\rho}_{x} \widehat{f}_{-x}\right\}\right\rangle_{0}=-\frac{1}{2}\left(\varepsilon_{\mathbf{q}}+\mathrm{i} \nu\right) \mu_{2}^{0}(x,-x),
$$

averages $\left\langle T\left\{B_{x} B_{-x}\right\}\right\rangle_{0},\left\langle T\left\{\widehat{f}_{x} \widehat{f}_{-x}\right\}\right\rangle_{0}$ are defined by (48). The symbol $\langle(\cdots)\rangle_{0}^{c}$ denominates the semi-invariant average over the states of free electrons and noninteraction plasmons.

The latter term in the exponent (64) is calculated in the same way

$$
(2 N)^{\frac{1}{2}} \beta^{-\frac{3}{2}} \alpha_{\mathbf{q}}^{-1}\left\langle T\left[\left(b_{x}+b_{-x}^{+}\right) \widehat{\rho}_{x} \mathrm{e}^{-\widehat{H}_{\mathrm{ep}}(\nu)}\right]\right\rangle_{0}^{c}=\frac{2}{\beta} \mu_{2}^{\mathrm{RPA}}(x,-x) .
$$

Similarly we calculate one term in the exponent of (64). In the ring diagrams approximation

$$
\begin{aligned}
\langle T & \left.\left\{\left(b_{x}+b_{-x}^{+}\right)\left(b_{-x}+b_{x}^{+}\right) \mathrm{e}^{-\widehat{H}_{\mathrm{ep}}(\nu)}\right\}\right\rangle_{0}^{c}=\left\langle T\left(b_{x}+b_{-x}^{+}\right)\left(b_{-x}+b_{x}^{+}\right) \mathrm{e}^{-\widehat{H}_{\mathrm{ep}}(\nu)}\right\rangle_{0} \\
& +\left(\frac{\hbar^{2}}{2 m}\right)^{2}\left(2 N \beta \alpha_{\mathbf{q}}^{2}\right)^{-1}\left\langle T\left\{\widehat{f}_{x} \widehat{f}_{-x}\right\}\right\rangle_{0}\left\langle T\left\{\left(b_{x}+b_{-x}^{+}\right) \widehat{B}_{-x}\right\}\right\rangle_{0} \\
& \times\left\langle T\left\{\widehat{B}_{x}\left(b_{-x}+b_{x}^{+}\right)\right\}\right\rangle_{0}\left\{1-\left\langle T\left[\widehat{H}_{\mathrm{ep}}^{2}(\nu)\right]\right\rangle_{0}^{\mathbf{q}, \nu}\right\}^{-1}
\end{aligned}
$$

therefore, one term of the exponent in the (64) is equal to $\beta^{-1} \sum_{\nu} \mu_{2}^{\mathrm{RPA}}(x,-x)$. Thus, the factor above $\varphi_{\mathbf{q}} \varphi_{-\mathbf{q}}$ is equal to zero in this approximation. So the two-electron structural factor

$$
S_{\mathbf{q}}^{\mathrm{RPA}}=(N \beta)^{-1} \sum_{\nu} \mu_{2}^{\mathrm{RPA}}(x,-x)
$$


was calculated over the operators $\widehat{\rho}_{x}$ or over the plasmon operators coinciding in the RPA. The consideration of higher diagrams in calculating the averages from (64) can give the factor above $\varphi_{\mathbf{q}} \varphi_{-\mathbf{q}}$ which is not equal to zero. Differently at the calculation of the $\langle\widehat{J}(b, a)\rangle_{\mathrm{ep}}$ we must take into account the terms which are proportional to the $\varphi_{\mathbf{q}_{1}} \varphi_{-\mathbf{q}_{1}} \varphi_{\mathbf{q}_{2}} \varphi_{-\mathbf{q}_{2}}$ or the other terms. These terms provide the coincidence of the integrals over the variables $\varphi_{\mathbf{q}}$. However, the basic contribution of the $\langle\widehat{J}(b, a)\rangle_{\mathrm{ep}}$ into thermodynamic potential is proportional to the $\beta^{-1}$. At low temperatures the contribution of the $\langle\widehat{J}(b, a)\rangle_{\text {ep }}$ in the energy of the system is equal to zero.

\section{Conclusions}

As it follows from the above stated matters, we can describe the electron liquid using the electron-plasmon model with Hamiltonian

$$
\widehat{H}=\widehat{H}_{\mathrm{a}}+\widehat{H}_{\mathrm{ep}}+\widehat{H}_{\mathrm{p}}+\widehat{H}_{\mathrm{pp}}
$$

This Hamiltonian describes two different subsystem objects: the electrons and plasmons which interact between each other (components of (70) are specified by the expressions (16), (22), (26)). The description of the electron liquid model in the electron and plasmon terms is completely equivalent to the traditional description in the particle terms with the Coulomb interaction, apparently the results from chapter 3 . At first glance a paradox situation arises since the model (70) describes the physical system with a higher degree of freedom than the traditional one

$$
\widehat{H}=\sum_{\mathbf{k}, s} \varepsilon_{\mathbf{k}} a_{\mathbf{k}, s}^{+} a_{\mathbf{k}, s}+(2 V)^{-1} \sum_{\mathbf{q} \neq 0} V_{\mathbf{q}}\left\{\widehat{\rho}_{\mathbf{q}} \widehat{\rho}_{-\mathbf{q}}-\widehat{N}\right\}
$$

However, under the conditions of thermodynamic equilibrium at low temperatures both models give equivalent results and thus the factor $\langle\widehat{J}(b, a)\rangle_{\mathrm{ep}}$ (see $(45)$ ) is of no importance here. We can explain this fact in the following way. In the conditions of thermodynamic equilibrium at low temperatures when the mean values of the occupation plasmon numbers are exponentially small quantities, we do not have to do with the real plasmons but with virtual plasmons. These plasmons (virtual) make the interaction between the electrons. Therefore, the real numbers of the degree of freedom coincided in the both models.

The perturbation theory over the operator of the electron-plasmon interaction does not have the divergent diagrams. To prove the exact equivalence of both models the summation series (non-divergent) of the diagrams is necessary to be done only for the weak non-ideality region. The series of the perturbation theory coincides well especially in the strong non-ideality range, as it is apparent from the results of the calculation of the ground state energy. In this connection, the summation of the infinite series of diagrams becomes unnecessary. Similar is the situation at the calculation of the structural factor of the model:

$$
S_{\mathbf{q}}=(2 \beta)^{-1} \sum_{\nu} \alpha_{\mathbf{q}}^{-2}\left\langle T\left(b_{x}+b_{-x}^{+}\right)\left(b_{-x}+b_{x}^{+}\right)\right\rangle_{\mathrm{ep}} .
$$


The first term in (68) gives the zero approximation over the operator $\widehat{H}_{\mathrm{ep}}(\nu)$,

$$
S_{\mathbf{q}}^{(0)}=\frac{\varepsilon_{\mathbf{q}}}{\hbar \omega_{\mathbf{q}}}\left(1+2 n_{\mathbf{q}}\right) \text {. }
$$

In the second order of the perturbation theory over the operator $\widehat{H}_{\mathrm{ep}}(\nu)$ we obtain

$$
S_{\mathbf{q}}^{(2)}=\beta^{-1} \sum_{\nu}\left[\left(\hbar \omega_{\mathbf{q}}\right)^{2}+\nu^{2}\right]^{-2}\left\{2 \varepsilon_{\mathbf{q}}\left(\left(\hbar \omega_{\mathbf{q}}\right)^{2}-\varepsilon_{\mathbf{q}}^{2}\right)+N^{-1} \mu_{2}^{0}(x,-x)\left[\nu^{2}+\varepsilon_{\mathbf{q}}^{2}\right]^{2}\right\} .
$$

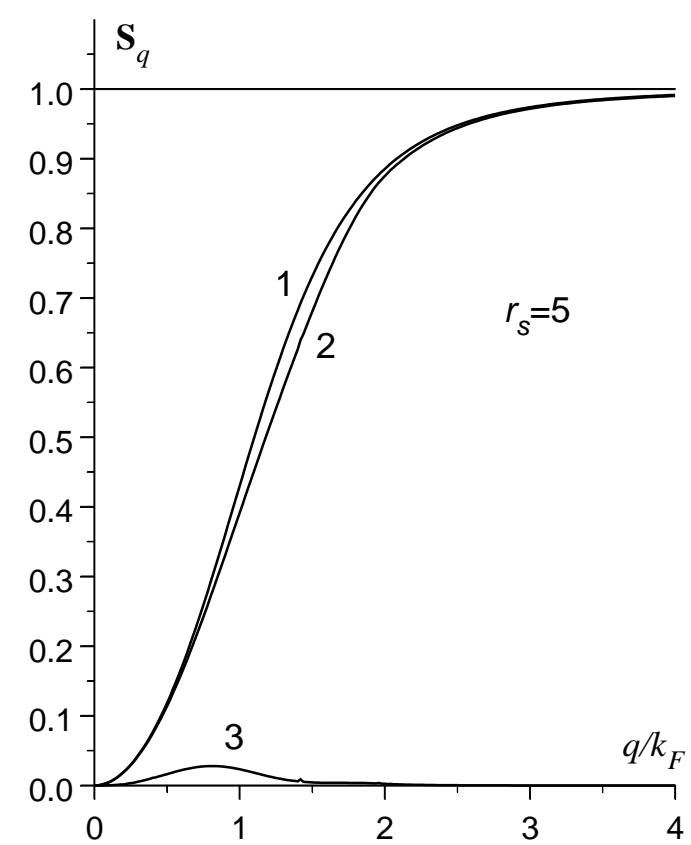

(a)

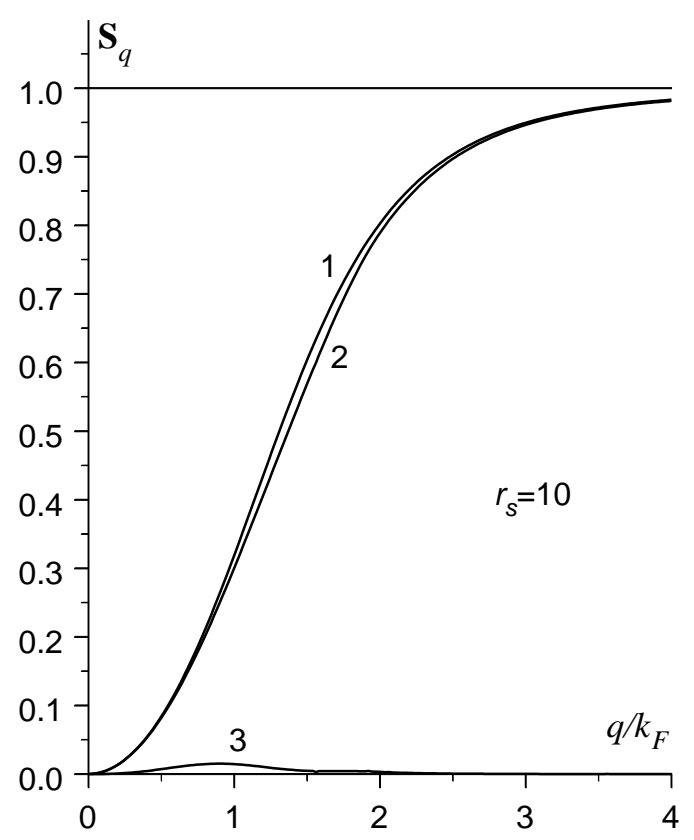

(b)

Figure 2. (a), (b): The structural factor of the electron liquid model in the ground state. Curve 1 corresponds to the zero approximation over the operator of electron-plasmon interaction. Curve 2 is the second order of the perturbation theory. Curve 3 is relative deviation $\left[S_{\mathbf{q}}^{R P A}-S_{\mathbf{q}}^{(2)}\right]\left[S_{\mathbf{q}}^{R P A}\right]^{-1}$.

The coincidence of the series diagrams of the perturbation theory at the calculation of structural factor is illustrated in figures $2 \mathrm{a}, 2 \mathrm{~b}$. In these figures the structural factor for $r_{s}=5$ and $r_{s}=10$ at the absolute zero of temperature is depicted. Curve 1 corresponds to approximation (73), curve 2 corresponds to (74), curve 3 is a relative deviation $\left[S_{\mathbf{q}}^{\mathrm{RPA}}-S_{\mathbf{q}}^{(2)}\right]\left[S_{\mathbf{q}}^{\mathrm{RPA}}\right]^{-1}$. The function $S_{\mathbf{q}}^{(0)}$ asymptotically (at $\mathbf{q} \rightarrow 0, \mathbf{q} \rightarrow \infty)$ coincides with $S_{\mathbf{q}}^{\mathrm{RPA}}$ which is apparent from the mentioned figures. The next terms of the formula (56) give only corrections. The relative deviation $S_{\mathbf{q}}^{(2)}$ from $S_{\mathbf{q}}^{\mathrm{RPA}}$ is approximately $4 \%$ in the maximum $\left(q \sim k_{\mathrm{F}}\right)$ at $r_{s}=5$. At $r_{s}=10$ the relative deviation is approximately $2 \%$.

The absence of the divergent diagrams and good coincidence of the series of perturbation theory over the operator of the electron-plasmon interaction make the electron-plasmon model actual for calculations of the characteristics of the electron liquid model in the strong non-ideality region. 


\section{References}

1. Bohm D., Pines D., Phys. Rev., 1951, 82, No. 4, 625-634.

2. Bohm D., Pines D., Phys. Rev., 1952, 85, No. 2, 338-353.

3. Bohm D., Pines D., Phys. Rev., 1953, 92, No. 3, 609-625.

4. Pines D., Phys. Rev., 1953, 92, No. 3, 626-636.

5. Bohm D., Huang K., Pines D., Phys. Rev., 1957, 107, No. 1, 71-80.

6. Nozieres P., Pines D., Phys. Rev., 1958, 111, No. 2, 442-454.

7. Pines D. Elementary excitations in solids. W.A.Benjamin, New-York, 1964.

8. Gell-Mann M., Brueckner K.A., Phys. Rev., 1957, 106, No. 2, 364-368.

9. Ceperley D.M., Alder B.J., Phys. Rev. Lett., 1980, 45, No. 7, 566-569.

10. Singwi K.S., Tosi M.P., Land R.H., Sjolander A., Phys. Rev., 1968, 176, No. 2, 589599.

11. Toigo F., Woodruff T.O., Ibid, 1971, 4, No. 2, 371-375.

12. Vashishta P., Singwi K.S., Phys. Rev. B, 1972, 6, No. 3, 875-887.

13. Emrich K., Zabolitzky J.G., Phys. Rev. B, 1984, 30, No. 4, 2049-2069.

14. Ichimaru S., Utsumi K., Phys. Rev. B, 1981, 24, No. 12, 7385-7388.

15. Yukhnovskii I.R., Ukr. Fiz. Zhurn., 1964, 9, No. 7, 702-714 (in Russian).

16. Yukhnovskii I.R., Ukr. Fiz. Zhurn., 1964, 9, No. 8, 827-838 (in Russian).

17. Yukhnovskii I.R., Tsiganenko V.V., Vavrukh M.V., Ukr. Fiz. Zhurn., 1965, 10, No. 2, 135-146 (in Russian).

18. Yukhnovskii I.R., Petrashko R.N., Teor. Mat. Fiz., 1973, 17, No. 1, 118-130 (in Russian).

19. Vavrukh M.V., Visnik Lviv. Univer. seriya fizichna, 1968, 3, 26-33 (in Ukrainian).

20. Feenberg E. Theory of quantum fluids. Academic, New-York, 1969.

21. Sinanoglu O., J. Chem. Phys., 1962, 36, No. 3, 706-717.

22. Sinanoglu O., Tuan D.F., J. Chem. Phys., 1963, 38, No. 7, 1740-1748.

23. Zubarev D.N., Dok. Akademii Nauk SSSR, 1954, 95, No. 4, 757-760 (in Russian).

24. Yukhnovskii I.R., Holovko M.F. The statistical theory of the classical equilibrium systems. Naukova dumka, Kiev, 1980 (in Russian).

25. Abramovitz M., Stegun I.A. Handbook of mathematical functions. National bureau of standards, USA, 1964 (in Russian).

26. Vavrukh M., Krokhmalskii T., Phys. Stat. Sol. (b), 1991, 168, 519-532.

27. Vavrukh M.V., Slobodyan S.B., Journ. Phys. Stud., 2003, 7, No. 3, 275-282 (in Ukrainian). 


\section{Електрон-плазмонна модель у теорії електронної рідини}

\section{М.В.Ваврух, С.Б.Слободян}

Львівський національний університет ім. І.Франка, кафедра астрофізики,

вул. Кирила і Мефодія, 8, 79005 Львів

Отримано 17 червня 2004 р., в остаточному вигляді 16 лютого 2005 р.

Запропоновано строгий підхід до опису моделі електронної рідини в термінах електронів і плазмонів. Концептуально наша робота близька до підходу колективних змінних, який розроблявся в роботах Бома і Пайнса. Проте ми використовуємо інший математичний апарат, в якому перехід до розширеного простору змінних частинок та плазмонів виконується за допомогою оператора переходу. Доведено, що у наближенні хаотичних фаз модель, яка складається із двох взаємодіючих між собою підсистем електронів і плазмонів, цілком еквівалентна моделі електронної рідини з кулонівською взаємодією.

Ключові слова: електронна рідина, плазмові коливання, кореляційна енергія, оператор переходу, структурний фактор

PACS: 05.30.Fk 